\title{
Renovated (nondual) approach to endometrial cancer typing: endocrine and inflammatory issues
}

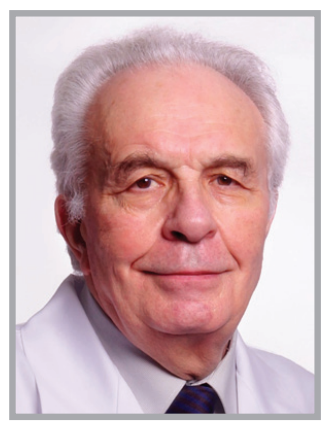

\author{
“...newer types of endometrial cancer may differ \\ by several characteristics other than molecular \\ genetics. They are probably very different in the \\ associated antitumor immune response and \\ tumor sensitivity or in resistance to hormones and \\ hormonal therapy."
}

\section{Lev M Berstein*}

First draft submitted: 23 August 2016; Accepted for publication: 30 September 2016; Published online: 10 October 2016

Endometrial cancer (EC) has lately become a focus of attention due to the rise in its incidence noted during the last decades [1] and the multitude of factors involved in its pathogenesis and progression pathways. Some of these factors are initiated in one way or another by hormonal stimuli. The most typical and widely known example is an estrogenic excess and progesterone deficit combined with involvement of endometrial receptors [2]. The second important group of factors discussed is metabolic disturbances (insulin resistance, glucose intolerance, hyperlipidemia and other metabolic syndrome components) [3,4], which are gradually becoming more prominent due to obesity epidemics and the shift in the ratio of certain obesity phenotypes [5]. Besides the hormonal (in particular, steroid) and metabolic components, there is an inflammatory component, which is presumed to act as the third important factor able to influence EC development [6]. The exact associations of these factors with rapidly changing basic conceptions on EC and with separate types of this tumor are still to be elucidated.
Notably, the pathogenetic classification of EC was traditionally built on dualistic conception with most attention devoted to whether or not hyperestrogenization is registered and endometrium hyperplasia or atrophy are present [7]. Starting from 1970 to the 1980s, the classification proposed by Bokhman became a dominating point of view. EC was divided into type I (70-75\% of cases) and type II (25-30\% of cases). The first type was more often seen in women with anovulatory uterine bleedings, late menopause, infertility, carbohydrate metabolism disorders and overweight. The tumor was usually highly or moderately differentiated and overall prognosis was good. In patients with EC type II, these characteristics were usually absent as most tumors were poorly differentiated and the prognosis was unfavorable [8]. Later on, mostly from 1990s, some authors (although they followed the same dualistic principle) started to somewhat modify this idea by confining the type I EC to endometrioid and type II EC to nonendometrioid tumors and presenting a relatively short list of characteristic mutations [7].

*Laboratory of Oncoendocrinology, NN Petrov Research Institute of Oncology, Pesochny-2, St Petersburg 197758, Russia; Tel.: +7 812439 9536; Fax: +7 812596 8947; levmb@endocrin.spb.ru

\section{KEYWORDS}

- endocrine and immune system $\bullet$ endometrial cancer - heterogeneity $\bullet$ immunotherapy and hormonal therapy $\bullet$ low-grade inflammation $\bullet$ mutations $\bullet$ obesity 


\section{"The continuation of research in this area may finally lead to more specifically individualized approach to endometrial cancer treatment and prevention, especially since the rate of this tumor incidence increase may vary significantly depending on endometrial cancer type."}

There has been a radical change in the last 3-4 years after results of EC tissue wholegenome sequencing were published. These results were included into The Cancer Genome Atlas and led to the conclusion for the need to distinguish between not just two types as previously thought, but a larger number of EC types [9-11]. The conclusions on EC molecularbased diversity were further somewhat specified in 2016 based on EC samples studies within the PORTEC trial [12].

As a result, some recent publications describe the detailed characteristic of at least four new EC variants, which were not known before $[10,11]$. These characteristics are suggested to be used for more precise disease course prediction while new versions of EC classification can be helpful in better understanding of this tumor pathogenesis and its variable sensitivity to treatment [11,13-14]. Among other things, the existing evidence suggests that more attention should be paid here to the EC-related proinflammatory and immune status [15].

According to The Cancer Genome Atlas data, which are currently discussed in several reports, the whole-genomic sequencing of endometrial carcinomas allowed allocation of four different cancer clusters based on POLE- $\varepsilon$ gene mutations. This gene codes for the DNA polymerase epsilon catalytic subunit involved in DNA synthesis and possibly excision repair. POLE gene mutations are found in $7-10 \%$ of endometrioid EC (cluster 1), and they are associated with higher incidence of other mutations ('ultramutator' phenotype) and another genetic abnormalities than in tumors from the remaining three clusters $[10,11]$. Cluster 1 (POLE- $\varepsilon$ mutated) and partially cluster 2 ECs (endometrioid tumors without $P O L E-\varepsilon$ mutations, but with microsatellite instability) demonstrate higher expression of genes involved in immune response compared with cluster 3 endometrioid cancers (no POLE- $\varepsilon$ mutations, no signs of microsatellite instability) and to cluster 4 (serous carcinomas of the endometrium) [15,16]. In particular, these genes code the expression of immune-checkpoint proteins, which filter and regulate signal intensity produced by or addressed to immune cells and include programmed death receptor ligands, PD-L1 and PD-L2. Currently, there are suggestions of trials employing PDL-targeted drugs already successfully used in melanoma and lung cancer (like pembrolizumab, Keytruda) - in EC patients with POLE- $\varepsilon$ mutations [15]. In the same cluster 1 carcinomas, an increased expression of T-cell markers (CD8A, PD-1) and a regulatory molecule CTLA- 4 has been revealed $[15,16]$, which in a way coincides with pre-existing data on intensive lymphoid infiltration of POLE- $\varepsilon$ mutated EC samples [13,17].

EC tumor tissue colonization by immune and para-immune cells (most often by lymphocytes and macrophages) can be viewed, inter alia, as a marker of so-called 'proinflammatory/genotoxic switch.' The signs of this switch are often observed in tumor and adipose tissue, for example, in breast cancer $[18,19]$. The proinflammatory/genotoxic switch association with different EC types undoubtedly requires further study due to the scarcity and incongruence of currently available data. Thus, proinflammatory cytokines such as TNF- $\alpha$ stimulate endometrial estrogens synthesis and, at the same time - their conversion to genotoxic derivatives [20]. Conversely, although some metabolic syndrome-associated characteristics often seen in EC patients (such as insulin resistance and overweight/obesity) are often accompanied by low-grade inflammation, these features do not always correlate with DNA damage or repair $[3,21]$. As a result, it is insufficient to compare tumor colonization by lymphocytes and macrophages in different EC types by focusing entirely on the data on POLE- $\varepsilon$ mutations and microsatellite instability $[13,17]$.

Significantly, some reports evaluate the correlation between macrophage/lymphocytes infiltration of EC tumor tissue and its receptor phenotype, in particular, the macrophage colonization correlated with loss of progesterone receptors [22]. While no less intensive tumor infiltration by FOXP3 ${ }^{+}$lymphocytes was seen mostly in estrogen receptor-negative tumors [23]. However, so far no correlation of this data to EC types (including recently described types) has been explored in depth.

Only a couple of current publications (in which EC cases were stratified based on POLE- $\varepsilon$ mutation status, presence of microsatellite instability, morphologic variant and differentiation grade) contained the data on steroid-receptor levels in tumor tissue. What is more, the published information is contradictory; in one paper, the progesterone receptors presence was characteristic for copy-number low, microsatellite stable endometrial carcinomas (cluster 3), 2\% of which were serous-like and $25 \%$ belonged to mixed type [10]; according to other observation, the loss of steroid receptors was mostly seen in 
serous $p 53$-mutant ECs (cluster 4) [12]. Although the latter phenomenon (receptor negativity of serous EC containing $p 53$ mutations) was often described before (see [7]), its occurrence was unexpectedly high in POLE-mutant tumors (cluster 1) [10,12], which do not correspond to their supposedly favorable prognosis $[17,24]$.

In conclusion, we should be reminded that currently nearly forgotten EC adjuvant therapy with progestins was widely used $30-35$ years ago [25]. This change in therapeutic approach may have promoted the switch of certain patient populations to apparently justified but harsher treatment regimens. However, recently obtained data point out the more pronounced heterogeneity of EC (the one of the most common cancers in female population) compared with previously common 'dichotomic' classification. As it was mentioned above, newer types of EC may differ by several characteristics other than molecular genetics. They are probably very different in the associated antitumor immune response and tumor sensitivity or in resistance to hormones and hormonal therapy. The continuation of research in this area may finally lead to more specifically individualized approach to EC treatment and prevention, especially since the rate of this tumor incidence increase [1] may vary significantly depending on EC type [26,27].

\section{Financial \& competing interests disclosure}

The manuscript has partial support of Russian Foundation of Basic Research (grant 15-04-00384 to LM Berstein). The author has no other relevant affiliations or financial involvement with any organization or entity with a financial interest in or financial conflict with the subject matter or materials discussed in the manuscript apart from those disclosed.

No writing assistance was utilized in the production of this manuscript.

\section{References}

1 Sheikh MA, Althouse AE, Freese KE et al. USA endometrial cancer projections to 2030 : should we be concerned? Future Oncol. 10(16), 2561-2568 (2014).

2 Kim JJ, Kurita T, Bulun SE. Progesterone action in endometrial cancer, endometriosis, uterine fibroids, and breast cancer. Endocr. Rev. 34(1), 130-162 (2013).

3 Berstein LM, Kvatchevskaya JO, Poroshina $\mathrm{TE}$ et al. Insulin resistance, its consequences for the clinical course of the disease, and possibilities of correction in endometrial cancer. J. Cancer Res. Clin. Oncol. 130 (11), 687-693 (2004).

4 Esposito K, Chiodini P, Capuano A, Bellastella G, Maiorino MI, Giugliano D. Metabolic syndrome and endometrial cancer: a meta-analysis. Endocrine 45(1), 28-36 (2014).

5 Berstein LM. Insulinemia, heterogeneity of obesity and the risk of different types of endometrial cancer: existing evidence. Expert Rev. Endocrinol. Metabol. 11(1), 51-64 (2016).

6 Dossus L, Lukanova A, Rinaldi S et al. Hormonal, metabolic, and inflammatory profiles and endometrial cancer risk within the EPIC cohort - a factor analysis. Am. J. Epidemiol. 177(8), 787-799 (2013).

7 Sherman ME. Theories of endometrial carcinogenesis: a multidisciplinary approach. Mod. Pathol. 13(3), 295-308 (2000).
8

Bokhman JV. Two pathogenetic types of endometrial carcinoma. Gynecol. Oncol. 15(1), 10-17 (1983).

9 Liang H, Cheung LW, Li J et al. Wholeexome sequencing combined with functional genomics reveals novel candidate driver cancer genes in endometrial cancer. Genome Res. 22(11), 2120-2129 (2012).

10 Cancer Genome Atlas Research Network, Kandoth C, Schultz N et al. Integrated genomic characterization of endometrial carcinoma. Nature 497(7447), 67-73 (2013).

11 Murali R, Soslow RA, Weigelt B. Classification of endometrial carcinoma: more than two types. Lancet Oncol. 15(7), e268-e278 (2014).

12 Stelloo E, Nout RA, Osse EM et al. Improved risk assessment by integrating molecular and clinicopathological factors in early-stage endometrial cancer-combined analysis of the PORTEC cohorts. Clin. Cancer Res. 22(16), 4215-4224 (2016).

13 Hussein YR, Weigelt B, Levine DA et al. Clinicopathological analysis of endometrial carcinomas harboring somatic POLE exonuclease domain mutations. Mod. Pathol. 28(4), 505-514 (2015).

14 Billingsley CC, Cohn DE, Mutch DG, Stephens JA, Suarez AA, Goodfellow PJ. Polymerase $\varepsilon$ (POLE) mutations in endometrial cancer: clinical outcomes and implications for Lynch syndrome testing. Cancer 121(3), 386-394 (2015).
15 Mehnert JM, Panda A, Zhong $\mathrm{H}$ et al. Immune activation and response to pembrolizumab in POLE-mutant endometrial cancer. J. Clin. Invest. 126(6), 2334-2340 (2016).

16 Gargiulo P, Della Pepa C, Berardi S et al. Tumor genotype and immune microenvironment in POLE-ultramutated and MSI-hypermutated endometrial cancers: new candidates for checkpoint blockade immunotherapy? Cancer Treat. Rev. 48, 61-68 (2016)

17 van Gool I, Eggink FA, Freeman-Mills L et al. $P O L E$ proofreading mutations elicit an antitumor immune response in endometrial cancer. Clin. Cancer Res. 21(14), 3347-3355 (2015).

18 Berstein LM, Kovalevskij AY, Poroshina TE $e t$ al. Signs of proinflammatory/genotoxic switch (adipogenotoxicosis) in mammary fat of breast cancer patients: role of menopausal status, estrogens and hyperglycemia. Int. J. Cancer 121(3), 514-519 (2007).

19 Howe LR, Subbaramaiah K, Hudis CA, Dannenberg AJ. Molecular pathways: adipose inflammation as a mediator of obesityassociated cancer. Clin. Cancer Res. 19(22), 6074-6083 (2013).

20 Salama SA, Kamel MW, Diaz-Arrastia CR et al. Effect of tumor necrosis factor-alpha on estrogen metabolism and endometrial cells: potential physiological and pathological relevance. J. Clin. Endocrinol. Metab. 94(1), 285-293 (2009). 


\section{EDITORIAL Berstein}

21 Postawski K, Przadka-Rabaniuk D, Piersiak T. 8-Oxo-7,8-dihydroguanine level - the DNA oxidative stress marker - recognized by fluorescence image analysis in sporadic uterine adenocarcinomas in women. Ginekol. Pol. 84(1), 44-50 (2013).

22 Jiang XF, Tang QL, Li HG et al. Tumorassociated macrophages correlate with progesterone receptor loss in endometrial endometrioid adenocarcinoma. J. Obstet. Gynaecol. Res. 39(4), 855-863 (2013).

23 Giatromanolaki A, Bates GJ, Koukourakis MI et al. The presence of tumor-infiltrating FOXP3+ lymphocytes correlates with intratumoral angiogenesis in endometrial cancer. Gynecol. Oncol. 110(2), 216-221 (2008).

24 McConechy MK, Talhouk A, Leung S et al. Endometrial carcinomas with pole exonuclease domain mutations have a favorable prognosis. Clin. Cancer Res. 22(12), 2865-2873 (2016).

25 Thornton JG, Ali S, O’Donovan P, Griffin N, Wells M, MacDonald RR. Flow cytometric studies of ploidy and proliferative indices in the Yorkshire trial of adjuvant progestogen treatment of endometrial cancer. Br. J. Obstet. Gynaecol. 100(3), 253-261 (1993).
26 Evans T, Sany O, Pearmain P, Ganesan R, Blann A, Sundar S. Differential trends in the rising incidence of endometrial cancer by type: data from a UK population-based registry from 1994 to 2006. Br. J. Cancer 104(9), 1505-1510 (2011).

27 Berstein LM, Poroshina TE, Turkevich EA et al. Features of endometrial cancer in patients with 'metabolically healthy' versus 'standard' obesity: the decreasing frequency of metabolically healthy obesity. Future Sci. OA 1(4), 1-11 (2015). 